\title{
X-RAY NOVA FLARES
}

\author{
P. R. AMNUEL and O. H. GUSEINOV \\ Shemakha Astrophysical Observatory of the Academy of Sciences, Azerb. S.S.R., U.S.S.R.
}

\begin{abstract}
In order to find out the physical nature of galactic X-ray sources, data on the variability of 24 sources during 1964-1971 have been investigated. The fluxes of 9 sources are found to be increasing to the maximum value (for several months) and then slowly decreasing (for 3 years). These 9 sources have been related by us to the class of X-ray Novae. The X-ray Nova synthetic light curve has been drawn from data of the fluxes of 9 discovered Novae. Assumptions have been made on the physical nature of the X-ray Novae. Between the flares the X-ray Novae may be weak X-ray sources with a luminosity of about $10^{34} \mathrm{erg} \mathrm{s}^{-1}$. During the flares the luminosity increases to about $10^{38} \mathrm{erg} \mathrm{s}^{-1}$. The number of $\mathrm{X}$-ray sources in the Galaxy is about $10^{3}-10^{4}$. The object of the optical identification may be a dwarf star of no earlier spectral class than $F$.
\end{abstract}

\section{Introduction}

Since the launching of the Uhuru satellite, our knowledge of galactic X-ray sources has considerably advanced. About $100 \mathrm{X}$-ray sources, mostly faint, were detected by Uhuru. Many of them are extra-galactic. The distribution of X-ray sources and their properties have been investigated by Amnuel and Guseinov $(1973,1974)$. It has been shown that there apparently exist two types of X-ray sources in the Galaxy: bright variable sources which are probably binary systems (Gursky, 1972; Shakura and Sunyaev, 1973) and weak constant sources whose nature remains obscure. According to Amnuel and Guseinov $(1973,1974)$ the average luminosity of bright variable X-ray sources is $\sim 10^{37}$ to $10^{38} \mathrm{erg} \mathrm{s}^{-1}$ if the average distance to them is $\sim 5 \mathrm{kpc}$. The average luminosity estimate of weak constant galactic sources is $\sim 10^{34} \mathrm{erg} \mathrm{s}^{-1}$ if the average distance is $\sim 1 \mathrm{kpc}$ (Amnuel and Guseinov, 1973). For both of these groups of sources, the average distance from the galactic plane $\overline{|z|}$ ranges from 250 to $460 \mathrm{pc}$. According to Amnuel and Guseinov $(1973,1974)$, the number of bright variable X-ray sources in the Galaxy is about $\mathbf{4 0}$ - practically all of them are now known. There should be many more weak constant X-ray sources; their estimated number is more than $10^{3}$.

\section{Selection of Observational Data}

The physical nature of variable $X$-ray sources is relatively clear: they appear to be binary systems with mass transfer onto a relativistic star (e.g., the sources Cyg X-1, Her X-1), while the nature of both strong and weak constant galactic X-ray sources, excluding supernova remnants, is obscure. To systematize data on the galactic X-ray sources and to investigate further their physical nature, a complete catalogue of all known galactic X-ray sources, which contains data beginning with the first rocket flights in 1964, has been compiled by Amnuel and Guseinov (1973). We have made use of the data from this catalogue as well as from the last Uhuru catalogue (Giacconi 
et al., 1974) in the present paper in an attempt to ascertain the nature of both strong and weak constant sources.

We have selected data for $24 \mathrm{X}$-ray sources from the catalogue by Amnuel and Guseinov (1973) for further consideration. At least two measurements of the flux before 1971 (i.e., before the launch of Uhuru) are given for each of them. Sixteen of these X-ray sources were also detected by Uhuru, but the other 8 , which had been previously detected, were not recorded during the Uhuru flight. The reasons for the disappearance of these 8 X-ray sources will be discussed later. Data on these 24 X-ray sources are very different - the observations were made at different times, in different energy intervals and by different instruments. Only for 15 of them can one find in the literature indications of a possible temperature of the bremsstrahlung spectrum but these temperature measurements are not very reliable.

To systematize the data, all the observed fluxes were reduced to the interval 2-6 $\mathrm{keV}$. We assumed that the spectrum of all the X-ray sources was bremsstrahlung; i.e.,

$$
\frac{\mathrm{d} N}{\mathrm{~d} E}=\frac{C}{E} e^{-E / k T},
$$

where $T$ is the temperature, $E$ is the photon energy and $N$ is the photon flux.

For the $15 \mathrm{X}$-ray sources with known temperature, we made use of the temperature to reduce the fluxes to the interval $2-6 \mathrm{keV}$. If the temperature of the $\mathrm{X}$-ray sources is not known, we used the following technique. We converted the given fluxes to the 2-6 $\mathrm{keV}$ interval for 10 different assumed values of the $\mathrm{X}$-ray source temperature in the 1-20 keV region (the temperatures of the majority of $X$-ray sources fall in this region), and then chose a temperature such that variations of the source brightness during the observations would be minimum. These temperature estimates approximately coincide with the temperatures indicated in the literature.

The choice of the minimum range made it necessary to search for constant flux for the $24 \mathrm{X}$-ray sources. However, it turned out that not one X-ray source could be assigned a constant flux no matter what temperature was chosen. Variability of the flux for any of the 24 sources was no less than a factor of 2 .

\section{Possible Types of Variability}

For $15 \mathrm{X}$-ray sources, no regularities in variation of brightness were found; a reliable light curve could not be drawn due to the lack of observations. As for the remaining $9 \mathrm{X}$-ray sources, they show quite a special type of variability and, most important, the shape of the light curve does not depend on the choice of temperature in the conversion to the 2-6 keV interval. The temperature in fact may be defined within a large error box since it will not have an effect on the type of variability. The latter fact indicates that the variability of these $9 \mathrm{X}$-ray sources has a real physical significance and is not a reflection of experimental or theoretical errors.

This special type of variability permits us to single out these $9 \mathrm{X}$-ray sources as a special class of sources. For 6 of the 9 sources, the increase of the flux towards maxi- 
mum was observed, after which it slowly decreased. Two of these 6 sources had weakened so much by 1971 that they were not detected by Uhuru. The other $4 \mathrm{X}$-ray sources were observed by Uhuru to be variable. However, whatever the type of variability, the range indicated by Giacconi et al. (1974) could not affect the general character of the light curves of these X-ray sources.

For three X-ray sources $(1725-30,1755-33$ and $2109+38)$, only a decrease of the flux was observed; for the X-ray sources $1725-30$ and $2109+38$, the decrease was so large that by 1971 they had disappeared. These three X-ray sources may be related to the same type as the first 6; the maximum fluxes from these X-ray sources might have been missed by earlier observers. The X-ray sources $1755-33$ and $2109+38$ reached maximum before 1965 when observations in the X-ray band were rare and unreliable. The X-ray source 1725 - 30 was first observable in 1969. In the same year it reached maximum; the maximum flux was hardly more than $0.2 \mathrm{cts} \mathrm{cm}^{-2} \mathrm{~s}^{-1}$. Indeed, if the maximum of this source were in 1968 or earlier and the flux were more than $0.2 \mathrm{cts}$ $\mathrm{cm}^{-2} \mathrm{~s}^{-1}$, then this X-ray source would have been observed by the rocket flight in December 1968 (Gorenstein, 1969) or in earlier measurements (see, for example, Meyer et al., 1970). These $9 \mathrm{X}$-ray sources have been assigned to the special class of the socalled Nova X-ray sources.

Four Nova X-ray sources were known before 1971: Cen X-2 was observable in 1967 (Harries, 1967; Rao et al., 1969), the flare of Cen X-4 occurred in the summer of 1969 (Evans et al., 1970) and the sources 1543-47 and 1735-28 flared in 1971 (Giacconi et al., 1974; Matilsky et al., 1972). The light curves of these Nova X-ray sources according to the above authors are shown in Figure 1. The source Cep X-4 which flared in June 1972 (Ulmer et al., 1973) is probably related to this class of X-ray sources. However, the variation in brightness of this source has been poorly investigated.

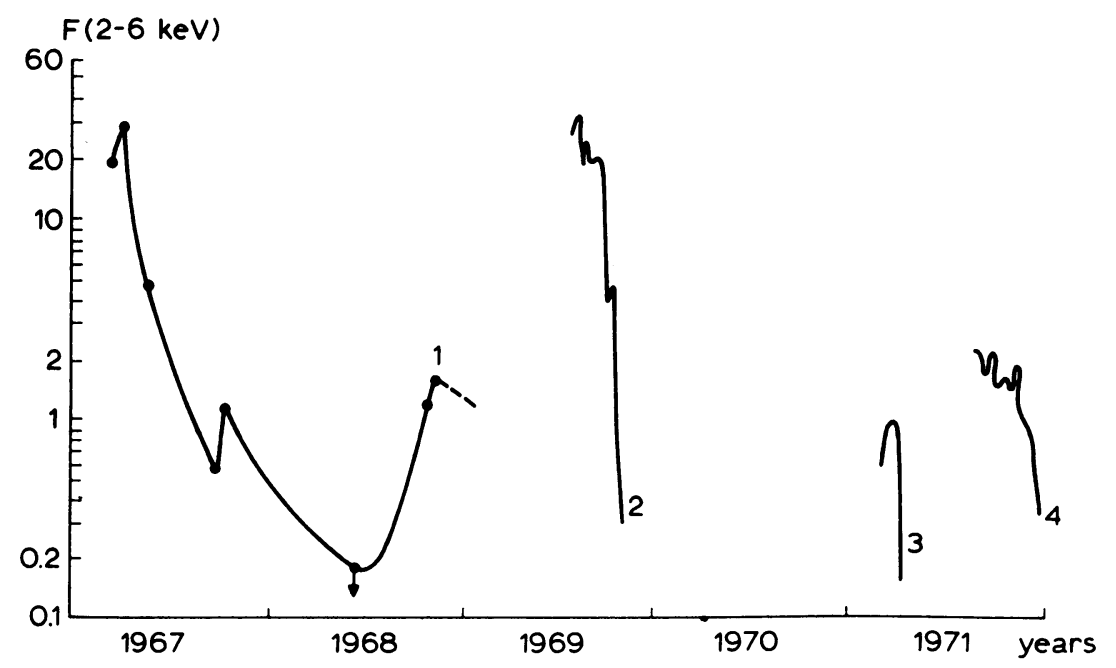

Fig. 1. Nova X-ray sources light curves. The sources are called: $1=$ Cen $X-2,2=$ Cen $X-4,3=$ $=1735-28$ and $4=1543-47$. 


\section{The Light Curve of Nova X-Ray Sources}

Certainly, it is impossible to draw a detailed light curve for each of the 9 Nova X-ray sources discovered by us, since none was observed more often than 5 times. However, one can draw a composite light curve for all of them.

For the unit of time, we take the time during which the flux from the sources decreases after maximum by a factor of 10 . For the unit of intensity, we take the intensity at maximum. This implies that the maximum brightness is reached when an $\mathrm{X}$-ray source is at zero phase, and when the phase is equal to one, the flux from the $\mathrm{X}$-ray source is equal to 0.1 of the maximum brightness. For each of the $6 \mathrm{X}$-ray sources for which a brightness maximum had been observed, the position of maximum on the time scale could be defined to within several months, and the value of maximum intensity to within $30 \%$ on average. As for the X-ray sources $1725-30,1755-33$ and $2109+38$, their time of and intensity at maximum could be defined with an accuracy good enough to include them in the composite light curve.

The composite light curve for the Nova X-ray sources drawn through 32 points is shown in Figure 2. For a comparison, the values for Cen X-4 are plotted on the same graph (crosses). All the measurements of the flux, including the Cen X-4 data, are in

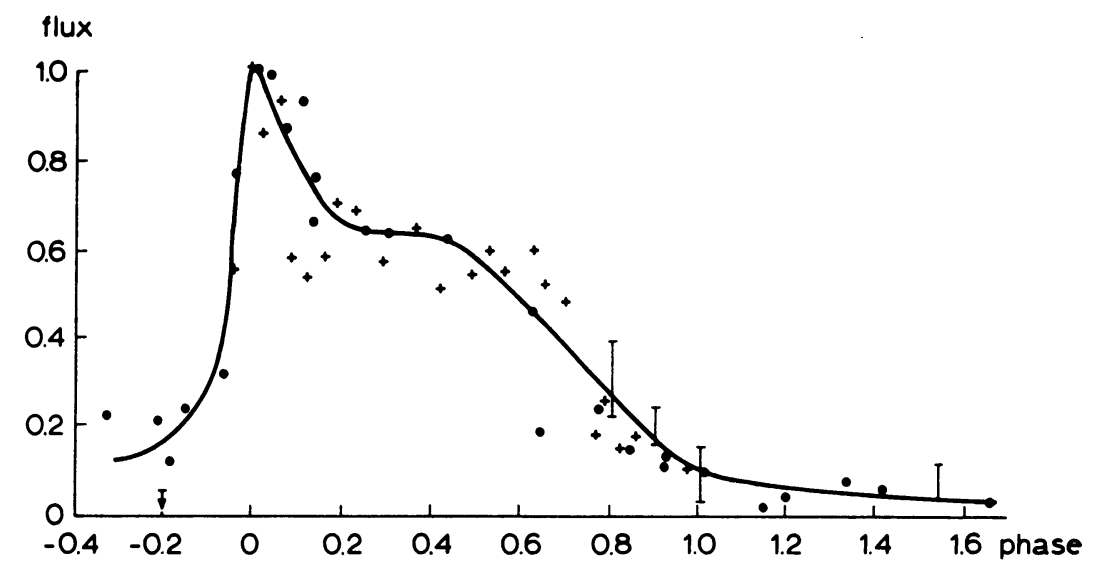

Fig. 2. The composite light curve for the Nova X-ray sources. Experimental data for the 9 Nova $\mathrm{X}$-ray sources are marked by filled circles. Data for Cen X-4 are marked by crosses.

good agreement with the general curve. As seen in Figure 2, the rapid increase of the flux (after phase -0.3 ) is followed by a rapid decrease to 0.6 flux units, after which (at phase 0.3 ) the flux remains almost constant; i.e., the curve has a plateau. The fluxes from the X-ray sources Cen X-4 and 3U 1543-47 have secondary maxima at phases $\sim 0.2$ and $\sim 0.6$. On the whole, the light curve of X-ray source $3 U 1543-47$ does not contradict the composite curve. The other Nova X-ray sources probably also have secondary maxima at the plateau in the composite light curve. The existence of the plateau in this case is explained by the different X-ray sources having secondary 
TABLE

Parameters of Nova X-ray sources

\begin{tabular}{|c|c|c|c|}
\hline $\begin{array}{l}\text { Nova X-ray } \\
\text { source }\end{array}$ & $\begin{array}{l}\text { The flux in } \\
\text { maximum } \\
\text { cts } \mathrm{cm}^{-2} \mathrm{~s}^{-1}\end{array}$ & $\begin{array}{l}\text { The period of } \\
\text { decrease of } \\
\text { the flux by a } \\
\text { factor two (unit }=1 \mathrm{yr} \text { ) }\end{array}$ & $\begin{array}{l}\text { The period of } \\
\text { decrease of } \\
\text { the flux by a factor } \\
10 \text { times (unit =1 yr) }\end{array}$ \\
\hline Cen X-2 & 28.0 & 0.04 & 0.19 \\
\hline Cen $X-4$ & 30.0 & 0.16 & 0.19 \\
\hline $1445-52$ & 1.0 & 1.2 & 2.8 \\
\hline $1543-47$ & 2.1 & 0.24 & 0.36 \\
\hline $1624-49$ & 0.7 & 3.2 & 5.8 \\
\hline $1655-42$ & 1.0 & 1.8 & 3.0 \\
\hline $1719-34$ & $\sim 0.5$ & 1.0 & 3.9 \\
\hline $1725-30$ & $>0.15$ & $\sim 0.8$ & $\sim 1.4$ \\
\hline $1728-16$ & 0.8 & 2.0 & 4.3 \\
\hline $1735-28$ & 1.0 & 0.05 & 0.08 \\
\hline $1755-33$ & $>0.4$ & $\sim 1.0$ & $\sim 3.9$ \\
\hline $1820-30$ & 1.3 & 3.8 & 6.8 \\
\hline $2109+38$ & $>0.2$ & $\sim 0.8$ & $\sim 3.3$ \\
\hline \multicolumn{2}{|c|}{ The average value } & 1.2 & 2.8 \\
\hline \multicolumn{2}{|c|}{$\begin{array}{l}\text { The average value for the } \\
\text { X-ray sources Cen X-2, Cen X-4, } \\
1543-47 \text { and } 1735-28\end{array}$} & 0.12 & 0.20 \\
\hline \multicolumn{2}{|c|}{$\left.\begin{array}{l}\text { The average value for the } \\
\text { remaining } 9 \text { Nova sources }\end{array}\right\}$} & 1.7 & 3.9 \\
\hline
\end{tabular}

moxxima at slightly different phases between 0.2 and 0.6 and/or by the inaccuracy in defining the phase for the $9 \mathrm{X}$-ray sources. It is impossible to draw light curves for Cen X-4 and 3U 1735-28 alone because of the lack of data.

After phase 0.6, a relatively drastic decrease in the flux to 0.15 units at phase 0.9 occurs and then a slower smooth lessening of the flux. On an average, it takes $2.8 \mathrm{yr}$ for the flux to decrease by a factor of 10 after maximum for all of the 13 Nova X-ray sources. Data on the characteristic time and average fluxes are listed in the table. The time for the flux increase before maximum is several times less.

\section{The Distribution in the Galaxy}

The average galactic latitude for the $12 \mathrm{X}$-ray sources (omitting the high-latitude source Cen X-4 which may be nearby) is $\overline{|b|} \approx 3$.7. In comparison, the average galactic latitude of variable X-ray sources is about $3^{\circ}$ and that of constant galactic $\mathrm{X}$-ray sources is about $7^{\circ}$ (Amnuel and Guseinov, 1974). Nova X-ray sources at maximum brightness belong to the strong sources (the average distance was estimated to be $\sim 5 \mathrm{kpc}$, according to Amnuel and Guseinov, 1974). The distribution of Nova X-ray sources in galactic longitude and their concentration in the direction of the Sco-Sgr arm show that the average distance to them is in fact $\sim 5 \mathrm{kpc}$. Then the average distance from the galactic plane is $|z| \approx 325 \mathrm{pc}$. Their luminosity at maximum is from $5 \times 10^{36}$ to $10^{38}$ 
$\operatorname{erg~s}^{-1}$; i.e., the same as the average luminosity of bright variable $\mathrm{X}$-ray sources (Gursky, 1972).

\section{The Physical Nature of Nova X-ray Sources}

At maximum brightness, a Nova X-ray source could be detected even if it were at the opposite side of the Galaxy. However, were the flux to weaken by several factors of 10 , then the X-ray source would become unobservable. Even if the luminosity of a Nova X-ray source at maximum is no less than $5 \times 10^{36} \mathrm{erg} \mathrm{s}^{-1}$, it will certainly be unobservable when its luminosity decreases to $5 \times 10^{34} \mathrm{erg} \mathrm{s}^{-1}$ (at the average distance of $\sim 5 \mathrm{kpc}$ ). Well below maximum brightness, a Nova X-ray source might have such a luminosity or even less; there are no observational data from which the conclusion might be drawn that, after the flare, X-ray emission completely stops. On the contrary, the character of the light curve (Figure 2) shows that the luminosity of X-ray sources at later phases decreases comparatively slowly which most likely amounts to a stationary value.

However, there are two classes of X-ray sources known to us (Amnuel and Guseinov, 1974): the first corresponds by luminosity $\left(10^{36}-10^{38} \mathrm{erg} \mathrm{s}^{-1}\right)$ to the Nova X-ray sources at maximum brightness, and the second $\left(\sim 10^{34} \mathrm{erg} \mathrm{s}^{-1}\right)$ to the same Nova $\mathrm{X}$-ray sources in their possibly stationary minimum state. Besides, as shown above, Nova X-ray sources are not separated spatially from the other two classes of X-ray sources. That is why it would be natural to try to unite bright variable, weak and Nova $\mathrm{X}$-ray sources on the basis of the assumption that all of them are X-ray sources of the same physical nature, but under different physical conditions.

The explanation of bright variable X-ray sources accepted nowadays (e.g. Gursky, 1972 ) is that they are binary systems with mass transfer onto a relativistic star. Bright constant X-ray sources, apparently, do not exist at all; this is confirmed by our sample of 24 X-ray sources. The number of bright X-ray sources amounts to $\sim 50$. This number includes those Nova X-ray sources listed by Giacconi et al. (1974). This means that most of the bright X-ray sources in the Galaxy are already known.

The number of weak X-ray sources (luminosity $\sim 10^{34} \mathrm{erg} \mathrm{s}^{-1}$ ) may amount to $10^{3}$ to $10^{4}$. If the difference between weak and strong $X$-ray sources lies only in the physical conditions of mass transfer onto a relativistic star, we have to suppose that the value of the accretion rate for the bright X-ray sources is larger by 3 to 4 orders of magnitude. Mass transfer onto a relativistic star in a binary system leads to the appearance of a gaseous disc of radius $\sim 10^{8}$ to $10^{9} \mathrm{~cm}$ emitting in the X-ray range(Shakura and Sunyaev, 1973).

Bright variable $\mathrm{X}$-ray sources in this case may be interpreted to be the same as flaring ones, but the accretion rate here is high, and consequently the period of mass accumulation in the disc is short. Such an X-ray source is in the 'active' phase practically all the time, and the amplitude of the flare here is no more than $\sim 5$ times the flux in the stationary state. Such a range has been indicated by Giacconi et al. (1974) for $1 / 3$ of all the variable $X$-ray sources.

Binary systems with a relativistic star and weak mass transfer are likely to be more 
widely spread. If luminosity of the disc in the stationary state is $\sim 10^{34} \mathrm{erg} \mathrm{s}^{-1}$, the number of such X-ray sources, according to Amnuel and Guseinov (1974), is $\sim 10^{3}$ to $10^{4}$.

So far between 1964 and 1971, at least 13 Nova X-ray sources have been discovered; the frequency of the occurrence of these flates (we should remember that we discovered almost all the Nova X-ray sources at maximum) is about $1.5 \mathrm{yr}^{-1}$ in the Galaxy. If the total number of such X-ray sources in the Galaxy is $\sim 10^{3}$ to $10^{4}$, then each of them would flare once in $\sim 10^{3}$ yrs. The disc in the stationary state might have a luminosity of $\sim 10^{33}$ to $10^{34} \mathrm{erg} \mathrm{s}^{-1}$ if the accretion rate were $\sim 10^{15} \mathrm{gm} \mathrm{s}^{-1}$. About $10^{25}$ to $10^{26} \mathrm{gm}$ might be accumulated in the disc between flares. Accretion of this quantity of matter onto the surface of a neutron star might provide a maximum luminosity of $\sim 10^{37} \mathrm{erg} \mathrm{s}^{-1}$ during a year.

The average distance to the weak $X$-ray sources observed at present is no more than $0.5 \mathrm{kpc}$. A supergiant at such a distance would be a first magnitude star. However, not a single weak X-ray source has been optically identified so far. It means that the companion of the degenerate star is no brighter than twelfth magnitude on average and differs little from other field stars. So it is easily seen why the weak X-ray sources have not been optically identified to date; the objects to be identified are not distinguished in any way. Dwarf stars suffer little mass loss which leads to small values of the flux and long intervals of time between the flares. Those few relativistic stars which have a supergiant companion constitute the bright X-ray sources of the Cyg X-1 type. On the other hand, a primary star may appear to be a flare star of the UV Cet type, in which case there appears an X-ray source of the Sco X-1 type indicated by Shklovsky (1973).

What is the likelihood of observing a flare of one of the known weak galactic X-ray sources in the near future? The number of such nearby X-ray sources now known is $10-20$, so one would expect to see a flare once every $50 \mathrm{yr}$. In order to record the flare of a distant weak X-ray source in the near future, counter sensitivity must be increased by approximately two orders of magnitude; then it will be possible to observe most of the weak X-ray sources throughout the Galaxy.

The Nova X-ray sources $1624-49,1719-34,1728-16,1755-33$ and 1820-30 observed in 1971 are expected to show, in future observations, a decrease in the flux until the source totally disappears.

There is the necessity to identify at least one weak X-ray source to ascertain their true nature. At present the identification of a weak galactic X-ray source is of greater importance than that of bright variable ones of the Cyg X-1 and Her X-1 type.

With further X-ray observations we expect to observe more flares of Nova X-ray sources. Observations of these X-ray sources in the active phase, confirmation of the detailed form of the light curve, and acquisition of spectra and of other physical parameters are necessary. These data will help us to better understand $\mathrm{X}$-ray sources and relativistic objects in the Galaxy. 


\section{References}

Amnuel, P. R. and Guseinov, O. H.; 1973, Variable Stars 19, 19; Astron. Tsirk, No. 795.

Amnuel, P. R. and Guseinov, O. H.: 1974, Astrofizika 10, 417.

Evans, W., Belian W., and Conner, J.: 1970, Astrophys. J. 159, L57.

Giacconi, R., Murray, S., Gursky, H., Kellogg, E., Schreier, E., Matilsky, T., Koch, D., and Tananbaum, H.: 1974, Astrophys. J. Suppl. 27, 237.

Gorenstein, P.: 1969, preprint, ASE-2297.

Gursky, H.: 1972, preprint, ASE-3066.

Harries, J.: 1967, Nature 215, 38.

Matilsky, T., Giacconi, R., Kellogg, E., and Tananbaum, H.: 1972, Astrophys. J. 174, L115.

Meyer, W., Bradt, H., and Rappaport, S. : 1970, Astrophys. J. 159, L115.

Rao, R., Chitnis, E., Prakasarao, A., and Jayanthi, U.: 1969. Astrophys. J. 157, L127.

Shakura, N. I. and Sunyaev, R. A.: 1973, Astron. Astrophys. $24,337$.

Shklovsky, I. S.: 1973, Astron. Zh. 50, 233 = Soviet Astron. A. J. 17, 157.

Ulmer, M., Baity, W., Wheaton, W., and Peterson, L.: 1973, Astrophys. J. 184, L117. 